\title{
Factores de riesgo relacionados a la falla de la sutura meniscal en niños y adolescentes
}

\section{Risk Factors Related to Meniscal Suture Failure in Children and Adolescents}

\author{
Rafael Calvo ${ }^{1,2}$ David Figueroa ${ }^{1,2}$ Paulina De La Fuente ${ }^{1,2}$ Daniel Paccot ${ }^{1} \quad$ R. Calvo Mena ${ }^{3}$ \\ Alex Vaisman ${ }^{1,2}$ Maria Jesus Tuca ${ }^{1,2}$ \\ ${ }^{1}$ Departamento de Traumatología y Ortopedia, Universidad del \\ Desarrollo, Santiago, Chile \\ 2 Departamento de Traumatología y Ortopedia, Clínica Alemana, \\ Santiago, Chile \\ ${ }^{3}$ Interno medicina Universidad del Desarrollo, Santiago, Chile \\ Address for correspondence Daniel Paccot, MD, Ladislao Errazuriz \\ 2028, dtpo 15, Providencia, Santiago, Chile \\ (e-mail: dpaccot@gmail.com).
}

\section{Resumen \\ Palabras Claves \\ - sutura meniscal \\ - niños \\ - pediatría \\ - lesión meniscal}

Las lesiones meniscales en niños son cada día más frecuentes. La preservación meniscal es una prioridad en este grupo de pacientes, sin embargo, existen pocos artículos que muestran los resultados de suturas meniscales en niños.

Objetivo El objetivo de este estudio es identificar factores de riesgo de falla en pacientes sometidos a una sutura meniscal en niños menores de 18 años.

Método Estudio retrospectivo de 35 pacientes sometidos a una sutura meniscal, con una edad promedio de 16 años (13-18) y un seguimiento de 71 meses de media (16115). Se evaluaron 6 variables: tipo, localización y tamaño de la lesión, edad, técnica quirúrgica y asociación a rotura de ligamento cruzado anterior (LCA). Los pacientes que presentaron falla de la sutura meniscal fueron identificados y sometidos a una regresión logística usando un modelo Stata V.14.0.

Resultados 9 pacientes (24.3\%) presentaron una falla en su cirugía durante el seguimiento. Lesiones iniciales mayores a $16 \mathrm{~mm}$ mostraron una tasa de re-rotura de un $73 \%$ independiente de la técnica quirúrgica. En lesiones menores a $16 \mathrm{~mm}$, se obtuvo un $78 \%$ de reparaciones exitosas. No se encontró relación entre la re-rotura meniscal y lesión de LCA, tipo y localización de la lesión, técnica quirúrgica y edad de los pacientes.

Conclusión Este estudio muestra que la reparación meniscal en niños tiene buenos resultados con una tasa de éxito promedio de un $75 \%$. El riesgo de falla de sutura meniscal se correlacionó con el tamaño inicial de la lesión con un $73 \%$ de fallo en lesiones mayores a $16 \mathrm{~mm}$ independientemente del tipo de cirugía.

Nivel de Evidencia 4, Serie de casos. received

January 12, 2017

accepted

March 20, 2017

published online

May 2, 2017
DOI https://doi.org/

$10.1055 / \mathrm{s}-0037-1602140$. ISSN $0716-4548$.
Copyright @ 2017 by Thieme Revinter

Publicações Ltda, Rio de Janeiro, Brazil
License terms

ㄷ) (i) $\ominus$ (\$) 
Abstract

\section{Keywords}

- meniscal suture

- children

- meniscal tear repare
Meniscal tears are uncommon in the pediatric population, with an increasing number. Currently meniscal preservation is a priority when treating these injuries. However, only a few studies have reported the clinical outcomes of arthroscopic meniscal repair in children and its risk factors of failure.

Objective Identify risk factors related to meniscal suture failure in patients under 18 years who underwent a meniscal repair.

Methods Retrospective study of 35 patients with an average age of 16 years ( $13-18)$ who underwent arthroscopic meniscal repair with a mean follow up of 71.1 months (16-115). We evaluate 6 variables: type, location and size of meniscal tear, age, surgical technique and anterior cruciate ligament (ACL) association. Patients with rerupture were identified and statistical analysis was performed through a logistic regression model using Stata V.14.0.

Results 9 patients (24.3\%) presented a suture failure during follow-up. Average time for re-rupture was 16 months (4-60 months). With an initial tear size of $16 \mathrm{~mm}$ or bigger, $73 \%$ of the meniscal repair will fail despite surgery technique. With an initial tear size smaller than $16 \mathrm{~mm}, 78 \%$ of will heal. No association was found between meniscal re-rupture and ACL rupture, type and location of tear, surgical technique and age. Conclusion: In our study meniscal repairs in pediatric population had good overall results with a global healing rate of $75.7 \%$. The risk of suture failure was related to the initial size of meniscal tear: when meniscal tear is bigger than $16 \mathrm{~mm}, 73 \%$ of them will fail despite surgery.

Level of Evidence 4, Case-series.

\section{Introducción}

Se ha reportado un claro aumento en la incidencia de las lesiones meniscales en pacientes jóvenes activos, posiblemente asociada a una mayor participación deportiva en esa población y una mejor capacidad diagnóstica tanto clínica como por imágenes. ${ }^{1,2}$ En niños mayores y adolescentes, aproximadamente el $80-90 \%$ de esas lesiones están en relación con la actividad deportiva y frecuentemente se asocian a otras lesiones agudas de la rodilla. ${ }^{3}$ Esta asociación es menos común en niños menores de 10 años, donde las lesiones se presentan generalmente en meniscos discoides. ${ }^{4,5}$

A pesar de todo eso, esa patología sigue siendo infrecuente en la población pediátrica con una incidencia aún desconocida. ${ }^{6}$ Stanitski et al estudiaron 70 niños (7-18 años) con hemartrosis traumática aguda de rodilla y hallaron lesiones meniscales en el 47\% de los preadolescentes (7-12 años) y en el $45 \%$ de los adolescentes (13-18 años). ${ }^{1}$

En las últimas décadas, diversos estudios han demostrado el importante rol del menisco en diversas funciones como estabilidad articular, transmisión de cargas, absorción de impacto y nutrición del cartílago articular. ${ }^{7}$ La meniscectomía total puede aumentar en un $235 \%$ el estrés de contacto de la articulación de la rodilla; resecciones por encima del $75 \%$ del menisco son equivalentes a meniscectomías totales al igual que la perdida de la raíz meniscal, por otra parte, resecciones menores no son inocuas pudiendo llegar aumentar hasta en un $65 \%$ ese estrés de contacto. ${ }^{5}$ Un estudio en niños demostró que después de una meniscectomía parcial o total, el 75\% de los pacientes quedaban sintomáticos y un $80 \%$ presentaban signos radiológicos de osteoartritis después de un seguimiento de 5.5 años. $^{5}$

El concepto de preservación meniscal es una prioridad en el tratamiento de las lesiones meniscales y se vuelve aún más indispensable en la población joven, donde la resección meniscal puede tener importantes consecuencias a largo plazo.

Hasta el día de hoy, sólo unos pocos estudios han informado sobre los resultados clínicos de las reparaciones meniscales por vía artroscópica en niños y adolescentes, demostrando mejores resultados que en suturas meniscales en adultos. ${ }^{1,8-10}$

El objetivo de este estudio es evaluar los resultados de las reparaciones meniscales en niños y adolescentes menores de 18 años e identificar los factores de riesgo en aquellos dónde se objetiva un fallo.

\section{Métodos}

\section{Pacientes}

Realizamos un estudio de cohorte retrospectivo en pacientes menores a 18 años sometidos a una reparación meniscal por vía artroscópica en nuestra institución, entre Junio de 2004 y Octubre de 2012. Los pacientes fueron identificados por la base de datos de la institución y la información fue recolectada desde los registros médicos. Los criterios de inclusión fueron: 18 años o menos, lesión meniscal de espesor total en zona roja en la resonancia magnética (RM) y seguimiento mínimo de 16 meses. Los criterios de exclusión fueron: 
cirugía previa en la rodilla, seguimiento incompleto, lesión de ligamento cruzado posterior o ligamento colateral, lesión osteocondral grado 3 y 4 en la artroscopia y fracturas periarticulares.

En cada caso se obtuvo de los registros médicos la siguiente información: edad, sexo, puntaje de Lysholm en el pre y postoperatorio, tipo de lesión meniscal durante la artroscopia (tamaño, morfología y ubicación) lesiones asociadas y técnica quirúrgica utilizada. Se consideró como una reparación exitosa, todos aquellos que presentaban una buena evolución clínica con ausencia de síntomas y signos meniscales y sin inflamación significativa. En casos donde se sospechó una re-rotura meniscal, se realizó un estudio imagenológico con artro-resonancia para confirmar el diagnóstico.

\section{Técnica Quirúrgica}

Los procedimientos quirúrgicos fueron realizados por dos cirujanos experimentados. Se realizó una artroscopia diagnóstica por portales artroscópicos clásicos en todos los casos, evaluando lesiones meniscales, ligamentosas y condrales.

Nuestras indicaciones para realizar un reparación meniscal fueron: lesiones meniscales sintomáticas ubicadas en el tercio periférico o zona vascular (zona roja-roja), lesiones verticales, mayores de $7 \mathrm{~mm}$ y con un tejido estructuralmente aceptable. Todas las lesiones fueron cruentadas con raspas, evaluando una reducción anatómica antes de realizar su fijación definitiva.

Los diferentes métodos e implantes utilizados para fijación en cada paciente, están detallados en la Tabla 1. El sistema de suturas híbridas más tornillos Clearfix ${ }^{\circledR}$ (Mitek, Norderstedt, Alemania), fueron preferidos en las lesiones donde estuvo comprometido el cuerno posterior, mientras que para las lesiones del cuerpo y del cuerno anterior se realizaron suturas convencionales (dentro-fuera y fuera dentro). El número de suturas e implantes utilizados para cada lesión, fue definido de acuerdo a la estabilidad lograda, considerando una longitud apropiada entre cada sutura/ implante entre $5-10 \mathrm{~mm}$.

En aquellos casos donde hubo lesión del LCA, se realizó simultáneamente su reconstrucción utilizando autoinjertos de isquiotibiales cuádruples. Solo un paciente presentó lesiones condrales asociadas, requiriendo microfracturas durante la intervención.

\section{Rehabilitación}

La rehabilitación postoperatoria incluyó movilización precoz con protección del rango de movimiento desde 0 hasta $70^{\circ}$, sin carga por las primeras 4 semanas y uso de férula de movimiento controlado. Luego se indicó un incremento paulatino de la carga y movilidad logrando un rango de 0 a $90^{\circ}$ con apoyo total a la semana 6 . Pivotear y hacer cuclillas no fueron permitidos hasta los 3-4 meses. El retorno gradual a las actividades deportivas de contacto se autorizó después de los 4-6 meses, dependiendo del progreso clínico, en los casos que se asoció a una reconstrucción del LCA ese se autorizó al $8^{\circ}$ mes. La indicación de una resonancia al 6 to mes, previo alta clínica, fue una preferencia del cirujano, conducta que no se aplicó a todos los pacientes del estudio.

\section{Análisis Estadístico}

Las variables continuas se describieron mediante promedio, desviación estándar y rango; fueron comparadas según rotura mediante el test t-Student. Las variables categóricas fueron descritas por frecuencias y proporciones; y comparadas por rotura mediante el test de independencia exacto de Fisher. Las variables cuya asociación con la rotura tuviesen una significación de 0.25 o menos, fueron retenidas para construir un modelo de regresión logística entre la rotura y esas variables. Dicho modelo fue depurado con un procedimiento de regresión paso a paso con probabilidad de retención del $p=0.05$. Las variables significativas resultantes del proceso de regresión fueron evaluadas en su capacidad prognóstica mediante el área bajo la curva ROC. Los intervalos de confianza son de un nivel de un 95\% y la significación fue del 5\%. Los datos fueron procesados como STATA versión 14.0.

\section{Resultados}

Treinta y cinco pacientes cumplieron con los criterios de selección, 9 mujeres (26\%) y 26 hombres (74\%), con un promedio de 16 años (13-18 años). Todas las lesiones meniscales se asociaron a traumatismos y ninguna estuvo relacionada a menisco discoideo. Como se detalla en la Tabla 1, las lesiones meniscales se localizaron principalmente en el menisco lateral (57\%) y menos frecuentemente en el menisco medial (43\%). La gran mayoría ocurrió en el cuerno posterior (74\%) o extendiéndose sobre el cuerpo meniscal (20\%). Los patrones de lesiones más comunes fueron el longitudinal (57\%) y asa de balde (31\%), siendo el menos común la lesión radial (9\%). El tamaño promedio fue de $16,2 \mathrm{~mm}$ (rango entre 7-30 mm). La media del puntaje de Lysholm preoperatorio fue de 76,7 . Veinte pacientes (57\%) presentaron una lesión concomitante del LCA, por lo que fueron sometidos a una reconstrucción simultánea de dicho ligamento en el mismo tiempo que la sutura meniscal. La técnica de reparación meniscal fue con suturas en 20 pacientes, tornillos en $11 \mathrm{y}$ ambos sistemas en 4 .

El promedio de seguimiento fue de 71,1 meses (16-115 meses) objetivándose 9 pacientes con una re-rotura, confirmada con artroresonancia y artroscopia. Esa rerotura se diagnsticó en promedio a los 16 meses post quirúrgico (4-60 meses). La tasa de recuperación fue de un 75,7\% durante el tiempo de seguimiento. La media del puntaje de Lysholm postoperatorio fue de 99,6 en el grupo de sutura meniscal considerada como exitosa frente a un 96,4 en el grupo de re-rotura, luego de una revisión artroscópica mediante menisectomía parcial. No se realizó una nueva sutura en los casos reoperados.

En la búsqueda por una asociación entre los estudios de variables individuales con una falla general en la reparación, se realizó un análisis estadístico (Tabla 2). De acuerdo al proceso paso a paso, sólo cuatro variables fueron propuestas para un modelo de regresión logística: tipo de rotura, tamaño 
Factores de riesgo relacionados a la falla de la sutura meniscal en niños y adolescentes Calvo et al. 37

Tabla 1 Datos de 35 pacientes

\begin{tabular}{|c|c|c|c|c|c|c|c|c|}
\hline Paciente & Edad & Tipo de lesión & Localización & Zona & Lesión asociada & $\begin{array}{l}\text { Tamaño } \\
(\mathrm{mm})\end{array}$ & Técnica & Reparación \\
\hline 1 & 15 & $\mathrm{~L}$ & 1 & PH & $\mathrm{ACL}$ & 15 & Tornillo & $\mathrm{s}$ \\
\hline 2 & 17 & $\mathrm{~L}$ & $E$ & $\mathrm{PH}$ & $\mathrm{ACL}$ & 15 & Sutura & $\mathrm{S}$ \\
\hline 3 & 15 & $\mathrm{~L}$ & 1 & $\mathrm{PH}$ & $x$ & 30 & Tornillo & $\mathrm{Rr}$ \\
\hline 4 & 18 & $\mathrm{BH}$ & I & $\mathrm{AH}$ & $\bar{x}$ & 25 & Sutura & $S$ \\
\hline 5 & 13 & $\bar{R}$ & 1 & $\mathrm{PH}$ & $x$ & 15 & Tornillo & $\mathrm{Rr}$ \\
\hline 6 & 15 & $\bar{L}$ & 1 & $\mathrm{PH}$ & $\overline{A C L}$ & 10 & Tornillo & $\mathrm{S}$ \\
\hline 7 & 14 & $\mathrm{~F}$ & $E$ & $\mathrm{PH}$ & $\mathrm{ACL}$ & 14 & Tornillo & $\mathrm{S}$ \\
\hline 8 & 14 & $\mathrm{~L}$ & $E$ & $\mathrm{PH}$ & $\mathrm{ACL}$ & 15 & Sutura & $\mathrm{S}$ \\
\hline 9 & 15 & $\mathrm{~L}$ & I & $\mathrm{PH}$ & $\mathrm{ACL}$ & 15 & Sutura + Tornillo & $S$ \\
\hline 10 & 18 & $\mathrm{BH}$ & 1 & $\mathrm{PH}-\mathrm{B}$ & $x$ & 20 & Sutura & $\mathrm{S}$ \\
\hline 11 & 15 & $\mathrm{~L}$ & $\mathrm{E}$ & $\mathrm{PH}$ & $\mathrm{ACL}$ & 15 & Sutura + Tornillo & $\mathrm{S}$ \\
\hline 12 & 15 & $\mathrm{~L}$ & $\mathrm{E}$ & $\mathrm{PH}$ & $\mathrm{ACL}$ & 20 & Sutura & $S$ \\
\hline 13 & 17 & $\bar{L}$ & 1 & $\mathrm{PH}$ & $\overline{A C L}$ & 12 & Tornillo & $\mathrm{S}$ \\
\hline 14 & 16 & $\mathrm{BH}$ & 1 & $\mathrm{PH}$ & $x$ & 20 & Sutura & $\mathrm{Rr}$ \\
\hline 15 & 17 & $\mathrm{BH}$ & $E$ & $\mathrm{PH}$ & $x$ & 20 & Sutura & $\mathrm{Rr}$ \\
\hline 16 & 16 & $\mathrm{~L}$ & $\mathrm{E}$ & $\mathrm{PH}-\mathrm{B}$ & $x$ & 20 & Sutura + Tornillo & $\mathrm{S}$ \\
\hline 17 & 18 & $\mathrm{BH}$ & 1 & $\mathrm{PH}$ & $\overline{\mathrm{ACL}}$ & 20 & Sutura + Tornillo & $\mathrm{Rr}$ \\
\hline 18 & 18 & $\mathrm{~L}$ & $E$ & $\mathrm{PH}$ & $\mathrm{ACL}$ & 8 & Sutura & $\mathrm{S}$ \\
\hline 19 & 16 & $\mathrm{~L}$ & $E$ & $\mathrm{PH}$ & $\mathrm{ACL}$ & 7 & Sutura & $\mathrm{S}$ \\
\hline 20 & 14 & $\mathrm{~L}$ & $E$ & $\mathrm{PH}$ & $x$ & 15 & Tornillo & $S$ \\
\hline 21 & 17 & $\mathrm{~L}$ & I & $\mathrm{PH}$ & $x$ & 15 & Tornillo & $\mathrm{s}$ \\
\hline 22 & 18 & $R$ & $\bar{E}$ & $\mathrm{PH}-\mathrm{B}$ & $\overline{A C L}$ & 10 & Tornillo & $\mathrm{S}$ \\
\hline 23 & 18 & $\mathrm{BH}$ & $E$ & $\mathrm{PH}$ & $x$ & 20 & Sutura & $S$ \\
\hline 24 & 17 & $\mathrm{~L}$ & 1 & $\mathrm{PH}$ & $\mathrm{ACL}$ & 15 & Tornillo & $S$ \\
\hline 25 & 17 & $\mathrm{BH}$ & $E$ & $\mathrm{PH}$ & $x$ & 10 & Tornillo & $\mathrm{s}$ \\
\hline 26 & 16 & $\mathrm{BH}$ & I & AH-B- $\mathrm{PH}$ & $x$ & 20 & Sutura & $\mathrm{S}$ \\
\hline 27 & 15 & $\mathrm{~L}$ & $\mathrm{E}$ & $\mathrm{PH}$ & $\mathrm{ACL}$ & 15 & Sutura & $\mathrm{Rr}$ \\
\hline 28 & 15 & $R$ & $\mathrm{E}$ & $\mathrm{PH}-\mathrm{B}$ & $\mathrm{ACL}$ & 15 & Sutura & $S$ \\
\hline 29 & 15 & $\mathrm{~L}$ & $E$ & $\mathrm{PH}-\mathrm{B}$ & $\mathrm{ACL}$ & 10 & Sutura & $\mathrm{s}$ \\
\hline 30 & 18 & $\mathrm{~L}$ & $E$ & $\mathrm{PH}$ & $\mathrm{ACL}$ & 15 & Sutura & $\mathrm{S}$ \\
\hline 31 & 15 & $\mathrm{BH}$ & $\bar{E}$ & $\mathrm{PH}-\mathrm{B}$ & $x$ & 20 & Sutura & $S$ \\
\hline 32 & 15 & $\mathrm{BH}$ & 1 & $\mathrm{PH}-\mathrm{B}$ & $x$ & 20 & Sutura & $\mathrm{Rr}$ \\
\hline 33 & 17 & $\mathrm{~L}$ & 1 & $\mathrm{PH}$ & $\mathrm{ACL}$ & 20 & Sutura & $\mathrm{Rr}$ \\
\hline 34 & 16 & $\mathrm{~L}$ & $E$ & $\mathrm{PH}$ & $\mathrm{ACL}$ & 10 & Sutura & $\mathrm{S}$ \\
\hline 35 & 18 & $\mathrm{BH}$ & $E$ & $\mathrm{AH}-\mathrm{B}$ & $x$ & 16 & Sutura & $\mathrm{Rr}$ \\
\hline
\end{tabular}

Abreviaciones: $\mathrm{AH}$, cuerno anterior; $\mathrm{B}$, cuerpo; $\mathrm{BH}$, bucket-handle; E, menisco externo; F, flap; I, menisco interno; L, longitudinal; LCA, ligamento cruzado anterior; PH, cuerno posterior; R, radial; Rr, Re-rotura; S, exitosa; X, sin lesión asociada.

de la lesión, localización y asociación con LCA, siendo el tamaño el único parámetro que demostró tener una asociación con el aumento de riesgo de re-rotura. Ese análisis demostró que todas las re-roturas fueron inicialmente de $16 \mathrm{~mm}$ o más, con una media de tamaño de lesión de $20 \mathrm{~mm}$ en ese grupo, mientras que en el grupo de éxito fue de $16 \mathrm{~mm}$ o menos. El gráfico (-Fig. 1) muestra el tamaño de las lesiones versus el éxito de las cirugías.

Se construyó una tabla que muestra la sensibilidad y especificidad que tiene el tamaño de la lesión para predecir los resultados de una cirugía (Tabla 3). Encontramos que con una lesión mayor o igual a $16 \mathrm{~mm}$, el porcentaje de fallo será 
38 Factores de riesgo relacionados a la falla de la sutura meniscal en niños y adolescentes Calvo et al.

Tabla 2 Asociación de variables individuales estudiadas con la falla de sutura meniscal

\begin{tabular}{|c|c|c|c|c|c|}
\hline \multirow[b]{2}{*}{ Categoría variable } & \multicolumn{2}{|l|}{ Exitosa } & \multicolumn{2}{|l|}{ Re-rotura } & \multirow[t]{2}{*}{ P-valor } \\
\hline & $\mathrm{N}$ & $\%$ & $\mathrm{~N}$ & $\%$ & \\
\hline \multicolumn{6}{|l|}{ Rotura } \\
\hline Asa de balde /flap & 7 & 26,9 & 5 & 55,6 & \\
\hline Longitudinal & 17 & 65,4 & 3 & 33,3 & \\
\hline \multirow[t]{2}{*}{ Radial } & 2 & 7,7 & 1 & 11,1 & \\
\hline & 26 & & 9 & & 0.16 \\
\hline \multicolumn{6}{|l|}{ Localización } \\
\hline Menisco externo & 17 & 65,4 & 3 & 33,3 & \\
\hline \multirow[t]{2}{*}{ Menisco interno } & 9 & 34,6 & 6 & 66,7 & \\
\hline & 26 & & 9 & & 0.129 \\
\hline \multicolumn{6}{|l|}{ Zona } \\
\hline Cuerno anterior & 1 & 3,8 & 0 & 0,0 & \\
\hline Cuerno posterior & 18 & 69,2 & 7 & 77,8 & \\
\hline \multirow[t]{2}{*}{ Cuerpo } & 7 & 26,9 & 2 & 22,2 & \\
\hline & 26 & & 9 & & 1 \\
\hline \multicolumn{6}{|l|}{ LCA concomitante } \\
\hline Sí & 17 & 65,4 & 3 & 33,3 & \\
\hline \multirow[t]{2}{*}{ No } & 9 & 34,6 & 6 & 66,7 & \\
\hline & 26 & & & 9,0 & 0.129 \\
\hline \multicolumn{6}{|l|}{ Técnica } \\
\hline Tornillo & 9 & 34,6 & 2 & 22,2 & \\
\hline Sutura & 14 & 53,8 & 6 & 66,7 & \\
\hline \multirow[t]{2}{*}{ Sutura + tornillo } & 3 & 11,5 & 1 & 11,1 & \\
\hline & 26 & & 9 & & 0.85 \\
\hline Variables continuas & Promedio & DS & Promedio & DS & \\
\hline Edad & 16.1 & 1.4 & 16 & 1.65 & 0.8397 \\
\hline Tamaño & 14.8 & 4.4 & 19.5 & 4.5 & 0.01 \\
\hline
\end{tabular}

de un $73 \%$ independiente del tipo de sutura. Por otro lado, una lesión inicial menor a $16 \mathrm{~mm}$, tendrá una tasa de éxito aproximada de un $78 \%$ independientemente de la técnica utilizada.

\section{Discusión}

Las lesiones meniscales en niños son a menudo suturadas, incluso en casos con bajas probabilidades de éxito. ${ }^{5-9}$ Sin embargo, no existen muchos estudios de resultados y factores de riesgo en ese grupo etario y una gran cantidad de datos se ha extrapolado de estudios en adultos.

Las suturas meniscales en niños y adolecentes han demostrado buenos resultados en la literatura. Vanderhave et al encontraron excelentes resultados clínicos en 95\% de sus pacientes con lesiones meniscales puras en menores de 18 años sin importancia en cuanto al tipo y temporalidad de la lesión. ${ }^{10}$ Krych et al obtuvieron un $74 \%$ de buenos resultados en pacientes menores de 18 años sometidos a una sutura meniscal, encontrando que esos resultados eran más favorables en lesiones más simples y en lesiones complejas que se acompañan de una lesión de LCA reparada. ${ }^{9}$ Noyes et al evaluaron los resultados en una población menor a 20 años sometida a reparación meniscal en zona roja-blanca logrando unos resultados favorables en un $75 \%$ a 4 años y $62 \%$ a 10 años. $^{11,12}$ En nuestro estudio de 35 pacientes menores de 18 años sometidos a reparación meniscal, obtuvimos resultados similares a la literatura con un $75 \%$ de buenos resultados.

Al realizar una regresión logística de nuestros casos de rerotura, el único parámetro que presentó una asociación positiva para una re-rotura meniscal, fue el tamaño inicial de la lesión, evidenciando que todas las re-roturas se produjeron en lesiones mayores a $16 \mathrm{~mm}$. Por lo demás, este análisis permitió evidenciar que la reparación de una rotura de más de $16 \mathrm{~mm}$, presentó un 73\% de fallas independientemente del tipo de sutura realizada. Finalmente, se objetivó que lesiones menores de $16 \mathrm{~mm}$, tenían un 78\% de resultados satisfactorios. Estudios previos han evidenciado esta misma 


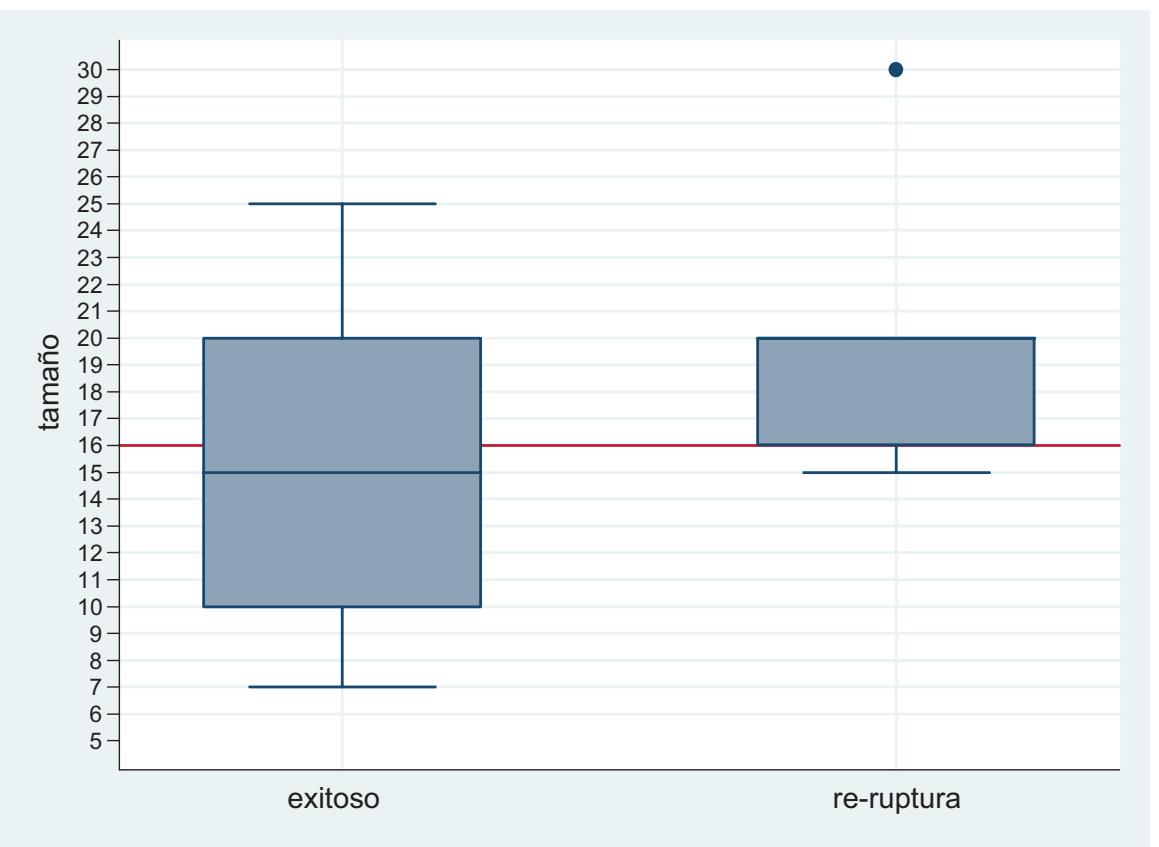

Fig. 1 Gráfico con tamaño de lesiones versus éxito de la cirugía.

asociación entre el tamaño de la rotura inicial y la tasa de fallo. Cannon et al describen que en pacientes con lesiones de menos de $2 \mathrm{~cm}$, la probabilidad de éxito es de $94 \%$, lesiones entre 2 y $4 \mathrm{~cm}$ presentan un $86 \%$ y las mayores de $4 \mathrm{~cm}$ solo un $50 \%{ }^{13,14}$ Publicaciones más recientes, como la de Haklar et al, han demostrado una asociación similar con buenos resultados en un $87 \%$ de los pacientes con lesiones de más de $20 \mathrm{~mm}$ y un $93 \%$ en lesiones de menos de $20 \mathrm{~mm} .{ }^{14} \mathrm{Si}$ bien algunos reportes de la literatura muestran malos resultados en lesiones de gran tamaño al igual que nuestro estudio, creemos que en niños es necesario reparar esas lesiones previo análisis y discusión con sus apoderados explicando la relevancia de preservar el menisco especialmente en ese tipo de pacientes .

En este estudio, un 57\% de los pacientes presentó una lesión de LCA asociada. Esa asociación en adolescentes es bastante común, y se considera que sólo un tercio de las lesiones meniscales son aisladas. ${ }^{10}$ Clásicamente las lesiones de LCA están asociadas a una lesión en asa de balde del menisco lateral. $^{1,6}$ Estudios previos han evaluado esta asociación encontrando que la lesión de LCA aumenta el potencial de cicatrización del menisco.,10,15 Kyrch et ali demostraron que en lesiones meniscales complejas, la asociación con lesión de LCA aumentaba la tasa de buenos resultados de 13 a $57 \%{ }^{9}$

Para eso se plantean tres teorías, la primera, es que las lesiones meniscales en rodillas inestables generalmente ocurren en zonas roja que tienen mayor potencial de cicatrización. En segundo lugar, se ha visto que lesiones longitudinales (que están asociadas a lesiones de LCA), mantienen la circunferencia de las fibras intactas y por lo tanto, presentan un mayor potencial de cicatrización. Finalmente, la lesión de

Tabla 3 Sensibilidad y especificidad de las re-roturas respecto a rotura inicial

\begin{tabular}{|c|c|c|c|c|c|}
\hline Corte & Sensibilidad & Especifici & & LR+ & $\mathrm{LR}_{-}$ \\
\hline$(\geq 7)$ & $100.00 \%$ & $0,00 \%$ & $25.71 \%$ & 1.0000 & \\
\hline$(\geq 8)$ & $100.00 \%$ & $3,85 \%$ & $28.57 \%$ & 1.0400 & 0.0000 \\
\hline$(\geq 10)$ & $100.00 \%$ & $7,69 \%$ & $31.43 \%$ & 1.0833 & 0.0000 \\
\hline$(\geq 12)$ & $100.00 \%$ & $26,92 \%$ & $45.71 \%$ & 1.3684 & 0.0000 \\
\hline$(\geq 14)$ & $100.00 \%$ & $30,77 \%$ & $48.57 \%$ & 1.4444 & 0.0000 \\
\hline$(\geq 15)$ & $100.00 \%$ & $34,62 \%$ & $51.43 \%$ & 1.5294 & 0.0000 \\
\hline$(\geq 16)$ & $77.78 \%$ & $73,08 \%$ & $74.29 \%$ & 2.8889 & 0.3041 \\
\hline$(\geq 20)$ & $66.67 \%$ & $73,08 \%$ & $71.43 \%$ & 2.4762 & 0.4561 \\
\hline$(\geq 25)$ & $11.11 \%$ & $96,15 \%$ & $74.29 \%$ & 2.8889 & 0.9244 \\
\hline$(\geq 30)$ & $11.11 \%$ & $100,00 \%$ & $77.14 \%$ & & 0.8889 \\
\hline$(>30)$ & $0.00 \%$ & $100,00 \%$ & $74.29 \%$ & & 1.0000 \\
\hline
\end{tabular}


LCA conlleva a la formación de un coágulo que trae células mesenquimáticas que favorecen la reparación. ${ }^{1}$

En nuestro estudio, no se encontró esa asociación entre lesión de LCA y los resultados de la sutura meniscal.

Existe un debate en la literatura respecto a la tasa de regeneración entre el menisco medial versus el lateral. Cannon et al describen que las reparaciones meniscales laterales tienen mejores resultados con respecto a las reparaciones mediales. ${ }^{13}$ Esa mayor falla en los meniscos mediales estaría dado por una mayor carga biomecánica (que son incluso mayores en concomitancia a una falla de LCA) y a un anclaje más firme. ${ }^{9}$ Sin embargo, en este estudio, no se encontró correlación entre el lado afectado y la tasa de re-rotura.

La asociación con otros factores de riesgo aparte de los mencionados previamente, no se han evidenciado en la literatura a pesar de haber sido estudiados. ${ }^{9-15}$ Solo un estudio de Krych et al obtuvo una correlación entre el tipo de lesión y los resultados. Ese estudio demostró que las lesiones meniscales simples lograron un $80 \%$ de buenos resultados, las en asa de balde desplazada un 68\%, y un $13 \%$ para las complejas, demostrando que el tipo de lesión inicial tiene una correlación directa con el resultado final. ${ }^{8}$ En nuestro estudio, no se evidenció una correlación significativa entre la re-rotura y el resto de los factores estudiados; edad, sexo, localidad y tipo de lesión.

Respecto a los métodos utilizados para la reparación meniscal, la evidencia es controversial. Haklar et al describen que la técnica de adentro hacia afuera tiene una tasa de recuperación significativamente superior comparada a la técnica todo dentro. ${ }^{14}$ Vanderhave et al también es partidario de la realización de una técnica de adentro hacia fuera para lograr mejores tasas de curación en niños. ${ }^{10}$ Por el contrario, Kyrch et al concluyen que las técnicas de fijación no tienen un efecto sobre el potencial de regeneración en las lesiones meniscales en niños ni adolescentes. ${ }^{9}$ En nuestro estudio, no encontramos diferencias significativas relacionadas al tipo de sutura. Probablemente esa controversia se deba a un bajo número de casos y a una amplia gama de opciones quirúrgicas, haciendo difícil una diferencia estadísticamente significativa.

Finalmente, consideramos como limitantes de nuestro trabajo, el ser un estudio retrospectivo y con una serie pequeña de pacientes. No todos los pacientes fueron sometidos a imágenes postoperatorias ni a un second look artroscópico que podría llevar a un sub-diagnóstico de las roturas.

\section{Conclusión}

La sutura meniscal en niños y adolescentes muestra buenos resultados generales, con una tasa de recuperación global de un $75,7 \%$

El tamaño de la lesión meniscal es determinante al pronosticar los resultados de la sutura. Lesiones meniscales mayores a $16 \mathrm{~mm}$ presentan una mayor tasa de fallo
Lesiones menores de $16 \mathrm{~mm}$ muestran los mejores resultados.

No se encontraron otros factores asociados al fallo ni tampoco una relación con lesión y reparación concomitante del LCA y mejores resultados de la sutura.

Creemos que es necesario estudios con mayor número de pacientes y mayor seguimiento para identificar nuevos factores de riesgo de falla de sutura meniscal.

\section{Bibliografía}

1 Kraus T, Heidari N, Švehlík M, Schneider F, Sperl M, Linhart W. Outcome of repaired unstable meniscal tears in children and adolescents. Acta Orthop 2012;83(03):261-266

2 Hantes ME, Kotsovolos ES, Mastrokalos DS, Ammenwerth J, Paessler HH. Arthroscopic meniscal repair with an absorbable screw: results and surgical technique. Knee Surg Sports Traumatol Arthrosc 2005;13(04):273-9

3 Shieh A, Bastrom T, Roocroft J, Edmonds EW, Pennock AT. Meniscus tear patterns in relation to skeletal immaturity: children versus adolescents. Am J Sports Med 2013;41(12):2779-2783

4 Andrish JT. Meniscal Injuries in Children and Adolescents: Diagnosis and Management. J Am Acad Orthop Surg 1996;4(05): 231-237

5 Kramer DE, Micheli LJ. Meniscal tears and discoid meniscus in children: diagnosis and treatment. J Am Acad Orthop Surg 2009; 17(11):698-707

6 Bellisari G1, Samora W, Klingele K. Meniscus tears in children. Sports Med Arthrosc Rev 2011;19(01):50-55

7 Terzidis IP, Christodoulou A, Ploumis A, Givissis P, Natsis K, Koimtzis M. Meniscal tear characteristics in young athletes with a stable knee: arthroscopic evaluation. Am J Sports Med 2006;34(07):1170-1175

8 Krych AJ, McIntosh AL, Voll AE, Stuart MJ, Dahm DL. Arthroscopic repair of isolated meniscal tears in patients 18 years and younger. Am J Sports Med 2008;36(07):1283-1289

9 Krych AJ, Pitts RT, Dajani KA, Stuart MJ, Levy BA, Dahm DL. Surgical repair of meniscal tears with concomitant anterior cruciate ligament reconstruction in patients 18 years and younger. Am J Sports Med 2010;38(05):976-982

10 Vanderhave KL, Moravek JE, Sekiya JK, Wojtys EM. Meniscus tears in the young athlete: results of arthroscopic repair. J Pediatr Orthop 2011;31(05):496-500

11 Noyes FR, Barber-Westin SD. Arthroscopic repair of meniscal tears extending into the avascular zone in patients younger than twenty years of age. Am J Sports Med 2002;30(04):589-600

12 Noyes FR, Chen RC, Barber-Westin SD, Potter HG. Greater than 10 -year results of red-white longitudinal meniscal repairs in patients 20 years of age or younger. Am J Sports Med 2011; 39(05):1008-1017

13 Cannon WD Jr, Vittori JM. The incidence of healing in arthroscopic meniscal repairs in anterior cruciate ligament-reconstructed knees versus stable knees. Am J Sports Med 1992;20(02):176-181

14 Haklar U, Donmez F, Basaran SH, Canbora MK. Results of arthroscopic repair of partial- or full-thickness longitudinal medial meniscal tears by single or double vertical sutures using the inside-out technique. Am J Sports Med 2013;41(03):596-602

15 Bach BR Jr, Dennis M, Balin J, Hayden J. Arthroscopic meniscal repair: analysis of treatment failures. J Knee Surg 2005;18(04): 278-284 